\title{
A FRAMEWORK TO GENERATE ACCURATE AND COMPLETE AS-BUILT BIMS BASED ON
} PROGRESSIVE LASER SCANS

\author{
* Te Gao ${ }^{1}$ and Burcu Akinci ${ }^{1}$ and Semiha Ergan ${ }^{1}$ and James H. Garrett, $\mathrm{Jr}^{1}$. \\ Department of Civil and Environmental Engineering \\ Carnegie Mellon University \\ 5000 Forbes Avenue \\ Pittsburgh PA, 15213 \\ (*Corresponding author: tgao@andrew.cmu.edu)
}




\title{
A FRAMEWORK TO GENERATE ACCURATE AND COMPLETE AS-BUILT BIMS BASED ON PROGRESSIVE LASER SCANS
}

\begin{abstract}
It is a general expectancy that in most of the construction and renovation project, the contractor is going to hand over the as-built documents to the owner or the facility management team at the completion of the project. The main challenge for the handover process is to ensure the completeness of the captured building information and the accuracy of it in terms of how well it represents the reality. Building information models (BIMs) can be used as an information repository to store and deliver as-built information. However, due to changes made in the construction and renovation phases and errors made in the design and modeling phases, discrepancies can exist between BIMs created in the design phase (asdesigned BIMs) and actual building conditions. Laser scanning technology is able to efficiently capture accurate geometric information, which provides an opportunity to identify and quantify discrepancies and update as-designed BIMs into as-built BIMs. This paper presents a case study, within which the asdesigned BIM of a newly renovated research lab was updated into an as-built BIM using laser scanned data captured in the renovation phase. This paper introduces the challenges associated with the updating process for the as-designed BIM. In order to address these challenges, this paper introduces a framework that supports the update of an as-designed BIM by incorporating point clouds captured by the progressive laser scans.
\end{abstract}

\section{KEYWORDS}

As-built BIMs, As-designed BIMs, Mapping, Discrepancy

\section{INTRODUCTION}

It is a general expectancy that in most of the construction and renovation project, the contractor is going to hand over the as-built documents to the owner or the facility management team at the completion of the project. In the current practice, changes that occurred in the construction or renovation phase are not always captured and updated to as-built documents in a consistent way (Pettee 2005, East and Brodt 2007, Fallon and Palmer 2007, Dickinson et al. 2009). According to a report published by NIST in 2004, $\$ 4.8$ billion is spent every year to ensure that the captured as-built information reflects the actual building conditions (Gallaher and Chapman 2004). As a digital representation that captures and exchanges building information between different stakeholders, building information models (BIMs) can be used as an information repository to store and deliver as-built information. In today's construction projects, asdesigned BIMs are created in the design phase based on design information. Although it is possible to convert an as-designed BIM into an as-built BIM, extensive surveying is needed since building projects usually have many changes during construction and renovation as project teams respond to change orders and unforeseen conditions (O'Brien 1998, Terwiesch and Loch 1999, Tang et al. 2010).

Laser scanning technology has the capability to efficiently capture the geometric information of a building in the form of point clouds. However, a point cloud is a collection of points with 3D spatial coordinates $(\mathrm{x}, \mathrm{y}, \mathrm{z})$, and it contains no semantic information (e.g., which building component that a point belongs to). Hence, in order to use geometric information contained in point clouds to update an asdesigned BIM, the current practice involves manually identifying and recognizing building components from point clouds and tracing points to determine the location and the dimension of building components. Since an as-designed BIM contains the semantic information associated with building components, there is a potential to map a point cloud to building components modelled in an as-designed BIM. Such mapping would be able to link the semantic information contained in an as-designed BIM together with a point 
cloud, so as to facilitate the process of recognizing and extracting building components from the point cloud.

The research presented in this paper targets addressing the challenges associated with the generation of as-builts aforementioned above and targets on understanding the challenges with and characteristics of the problem of updating an as-designed BIM based on point clouds captured by laser scans. We conducted a case study, within which the as-designed BIM of a newly renovated research lab was updated into an as-built BIM using point clouds captured progressively in the renovation phase. This paper presents the case study and the challenges associated with the process of updating an as-designed BIM into an accurate and complete as-built BIM based on point cloud data. To address the identified challenges, this paper introduces a vision for a framework that supports the process of updating an asdesigned BIM by incorporating the geometric information captured by laser scans frequently taken throughout the construction or renovation phases.

\section{AN OVERVIEW OF THE CASE STUDY}

A case study was conducted on a renovation project in a 100 year old university campus building. In this project, three adjacent rooms were renovated to accommodate a high-tech research lab. The scope of this renovation project included complete demolition of the interior of rooms and installation of new HVAC, gypsum wallboard, windows, drop ceilings, light fixtures, doors, and flooring. At different times during the renovation process, we visited the renovated space and took multiple laser scans that capture the interior of the research lab.

In the case study, we went through four steps for updating the as-designed BIM into the as-built BIM using point clouds. The first step is to segment a point cloud into segments (e.g., geometric primitives with closed boundaries or surfaces). After segmenting a given point cloud, the next step was to map the segments of the point cloud to the building components modeled in the as-designed BIM. The goal of this step was to identify the correspondences between the as-designed BIM and the actual building conditions captured by laser scans. Through the mapping process, the semantic information (e.g., object ID, object name) contained in the as-designed BIM could be linked to the segments of the point cloud. This linkage supported the further comparison of the same building components captured by the two data sources (i.e., point clouds versus as-designed BIMs). The third step was to compare the geometric information captured by the point cloud and the as-designed BIM and quantify the discrepancies between the two data sources. In the last step, the identified discrepancies were removed from the as-designed BIM so that the asdesigned BIM was updated to reflect the actual building conditions.

\section{IDENTIFIED CHALLENGES ASSOCIATED WITH THE UPDATING PROCESS}

Based on the case study, two challenges were identified in relation to the process of updating an as-designed BIM. First, the discrepancies between a point cloud and an as-designed BIM make it challenging to map the two data sources together. Second, laser scans performed at a single point in time only presents a partial view of the building and hence does not necessarily provide all of the geometric information needed to update of the as-designed BIM. This paper will mainly focus on the first challenge. The details of the second challenge can be found in Gao et al. (2012).

\section{Discrepancies between a point cloud and an as-designed BIM}

In the mapping step, segments of a point cloud are mapped to components modeled in an asdesigned BIM. The mappings can be identified by reasoning with various features of the point cloud and the BIM. For example, if segment $P_{i}$ of a point cloud is located at the same place where component $B_{j}$ is modeled in a BIM, then it is possible that $\mathrm{P}_{\mathrm{i}}$ and $\mathrm{B}_{\mathrm{j}}$ are the same component. However, a building component might have different shapes, dimensions, and be located at different positions in a point cloud as compared to how it is modeled in an as-designed BIM. These discrepancies could mislead the mapping algorithms and generate incorrect mappings between the point cloud and the BIM. 
Discrepancies may exist between a point cloud and an as-designed BIM because of the following reasons: (a) various types of changes (e.g., location, composition, geometry, etc.) in components made in the construction or renovation phase; (b) errors made in the as-designed BIM during the design and modeling phase; and (c) occlusions existing in a scene that prevent a laser scanner from capturing a complete view of building conditions. In the case study, we have identified five types of discrepancies between the point cloud and the as-designed BIM, which are: (a) Shape discrepancy, within which a component is constructed with a shape different than the shape specified in the as-designed BIM; (b) Location discrepancy, within which a component is constructed at a different location as compared to what is shown in the as-designed BIM; (c) Dimension discrepancy, within which the dimension of a component specified in the as-designed BIM is different than the dimension represented in the point cloud; (d) Content discrepancy, within which a component modeled in the as-designed BIM is not shown in the point cloud, and vice versa; and (e) Composition discrepancy, where a single component is modeled as a group of separate components in the as-designed BIM, and vice versa. Examples of such discrepancies are shown in Figure 1.
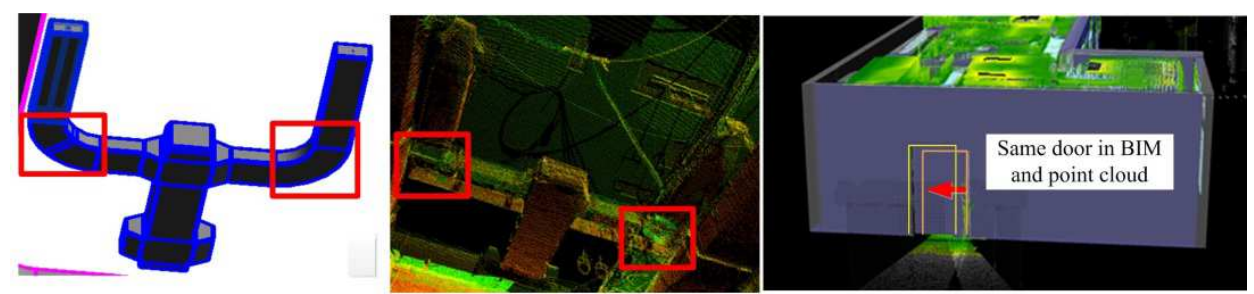

a. an example showing shape discrepancy in the air duct section analyzed in the case
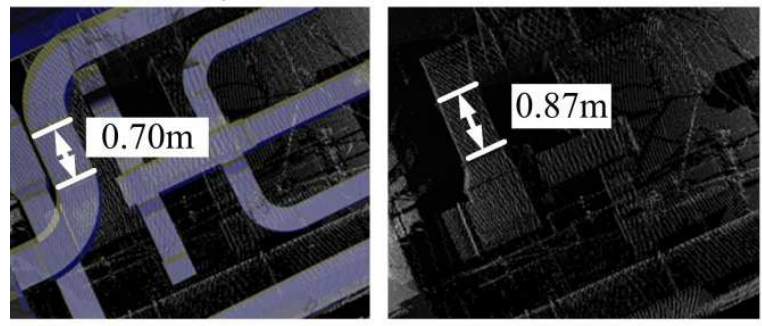

b. an example showing location discrepancy in the door analyzed in the case

C. an example showing dimension discrepancy in the air duct section analyzed in the case
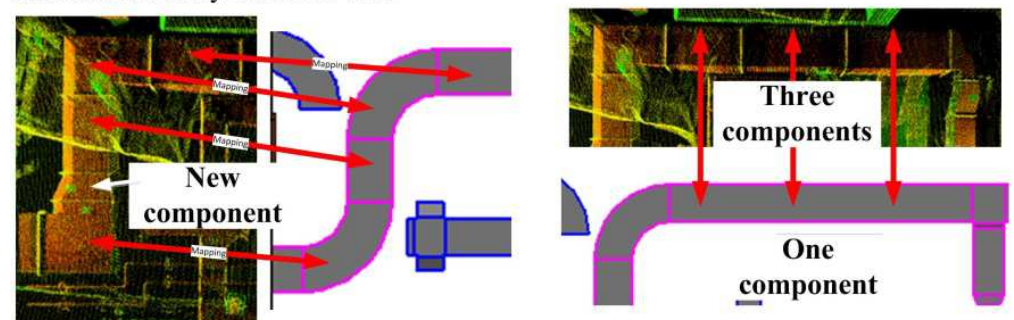

d. an example showing content discrepancy in the air duct section e. an example showing composition discrepancy in the air duct section analyzed in the case analyzed in the case

Figure 1 - Examples of different types of discrepancies identified in the case study

\section{Discrepancies impact the accuracy of mapping between an as-designed BIM with a point cloud}

To demonstrate the impacts of the discrepancies on the mapping step, in this paper we selected HVAC duct work installed in the case study project as the target components, and mapped the duct work from the point cloud to the as-designed BIM. In this mapping, the point cloud has been segmented and the segments are labeled as (P1, P2 ... P16), as shown in Figure 2. The duct works modeled in the as-designed $\mathrm{BIM}$ are labeled as (B1, B2... B16). 

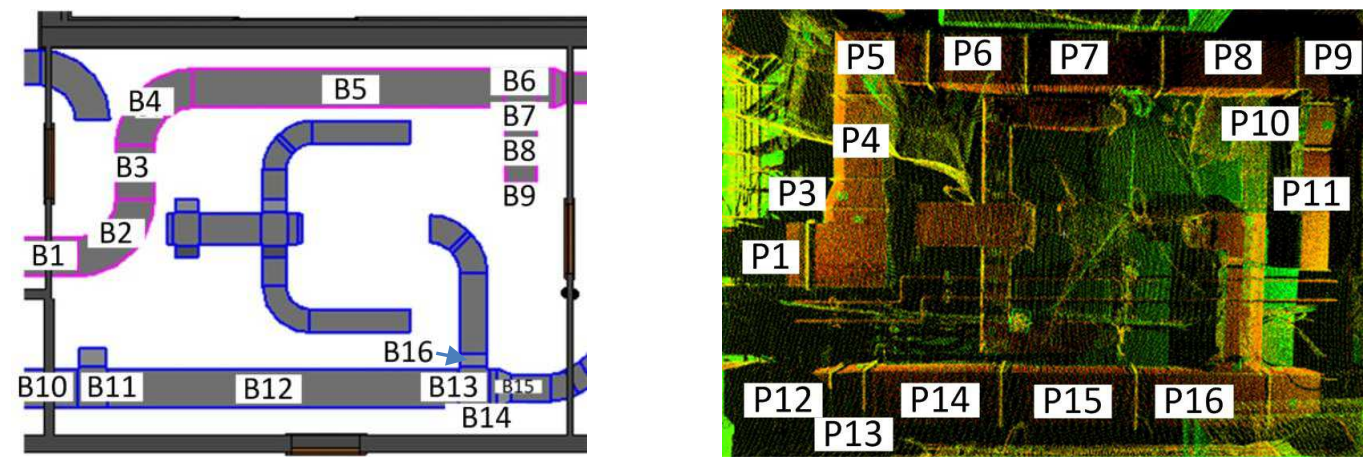

Figure 2 - The targeted air ducts shown in the as-designed BIM and the point cloud

Three different mapping approaches were tested in this mapping experiment, which were (a) spatial proximity-based mapping approach, (b) shape similarity-based mapping approach and (c) topologybased mapping approach.

\section{Spatial proximity-based mapping approach}

Possible mappings between a point cloud and a BIM can be identified by reasoning with their spatial proximity. The spatial proximity between a point cloud and a BIM can be calculated by projecting the registered point cloud and BIM into a $2 \mathrm{D}$ plane, and then reasoning about the overlapped surface area between the segments in the point cloud and components in the BIM. Figure 3 shows a ground projection of the registered point cloud and as-designed BIM. In the spatial proximity-based mapping approach, if the projected surface of segment $P_{\mathrm{i}}$ in the point cloud overlaps with the projected surface of component $B_{j}$ in the as-designed BIM, then it is likely that $P_{i}$ and $B_{j}$ are the same components and should be mapped to each other. If the segment $P_{\mathrm{i}}$ is spatially overlapped with multiple components $\left(B_{j}, B_{j+1}, B_{j+2} \ldots\right), P_{\mathrm{i}}$ is mapped to all these components.
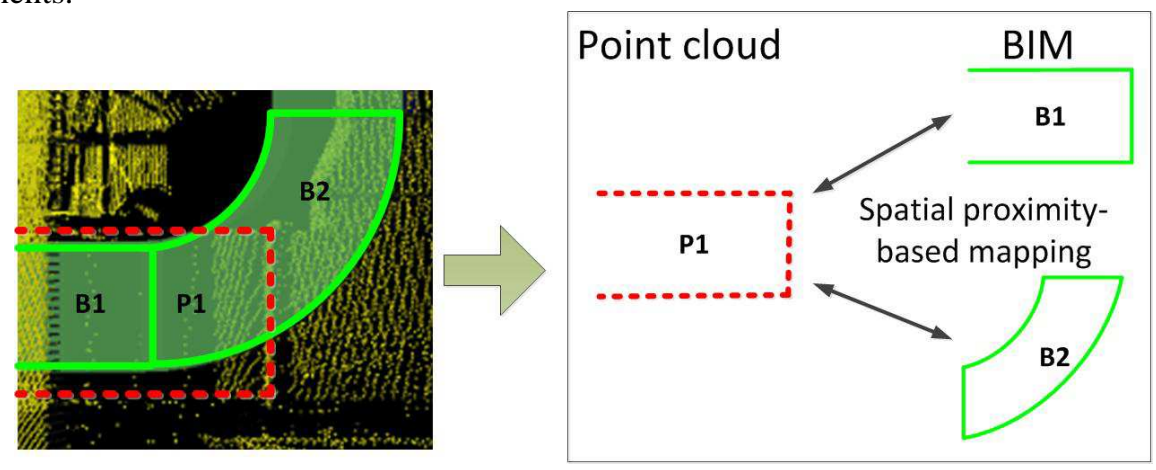

Figure 3 - Examples of the spatial overlaps between a point cloud and a BIM

$\underline{\text { Shape similarity-based mapping approach }}$

Mappings between two components in a point cloud and a BIM can be identified based on their shape similarities. For example, if segment $\mathrm{P}_{\mathrm{i}}$ of a point cloud has a similar shape as compared to component $B_{j}$ in a BIM, then it is possible that $P_{i}$ and $B_{j}$ are the same component. Since there is no standard way to define the 3D shape of a component, we decomposed the 3D shape of air ducts into two features, which are the cross section and the principle axis, and compared these two features in the 2D space. Typically, the cross sections of ductworks are modeled as regular polygons (i.e., square, trapezium and rectangle) and the principle axes of ductworks are modeled as a straight line, a curve line with right angle and a curve line with rounded angle. Hence, we defined the shape similarity between $P_{i}$ and $B_{j}$ as: 
- The cross sections of $\mathrm{P}_{\mathrm{i}}$ and $\mathrm{B}_{\mathrm{j}}$ have the same shape type, and the maximum dimension deviation is smaller than $\alpha$. The principle axes are modeled in the same type (i.e., straight line, curve line with right angle or curve line with rounded angle). The maximum deviation between the length of principle axis is smaller than $\beta$.

- $\alpha$ and $\beta$ are the thresholds to eliminate the impacts of the dimension discrepancies on the shape similarity-based mapping approach. If $\alpha$ and $\beta$ are set to zero, then the mapping approach is not tolerant to any dimension differences. In this experiment, we initially set $\alpha=6$ inches and $\beta=30$ inches to introduce a certain level of tolerance for the dimension differences into the mapping approach.

One major problem with the shape similarity-based mapping approaches is the large search space. To identify the corresponding BIM components for each segment in the point cloud, the approach needs to search for all the components modeled in the BIM. Assuming that there are $\mathrm{n}$ segments fitted to the point cloud and $\mathrm{m}$ components modeled in the as-designed BIM, the complexity of the search space is approximately $\mathrm{m}^{\mathrm{n}}$. In order to narrow down the search space, we combined the spatial proximity-based and shape similarity-based approaches together. As shown in Figure 4, we first applied the spatial proximitybased mapping approach to identify an initial set of mapping candidates, and then further filtered the mapping candidates based on their shape similarities.

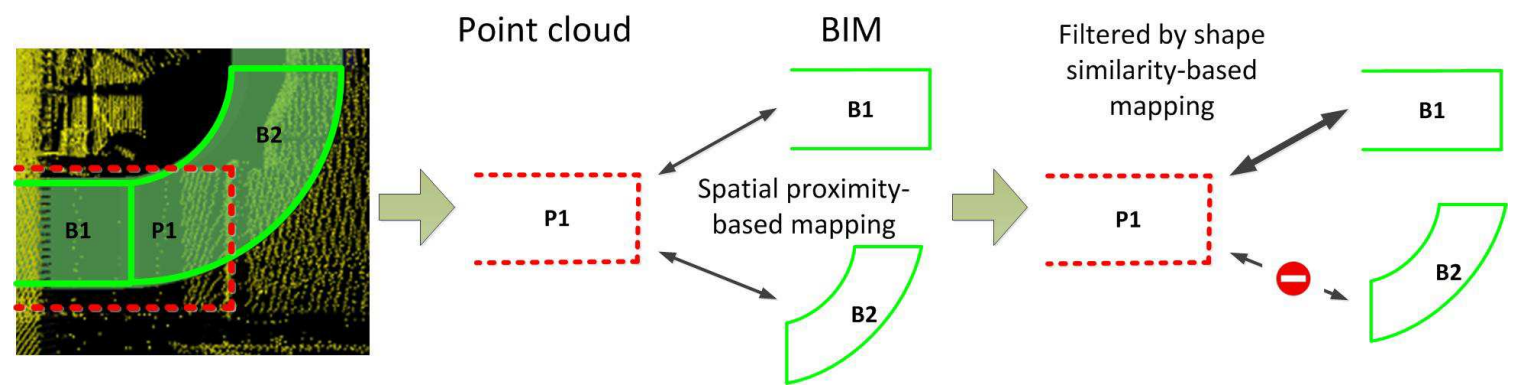

Figure 4 - An example of combining the shape similarity and spatial proximity-based mapping approaches

\section{$\underline{\text { Topology-based mapping approach }}$}

The mapping can also be identified by reasoning with the topological features of the point cloud and the as-designed BIM. The topological relationships of components captured by the point cloud and the as-designed BIM can be extracted and represented as a graph structure, as shown in Figure 5.

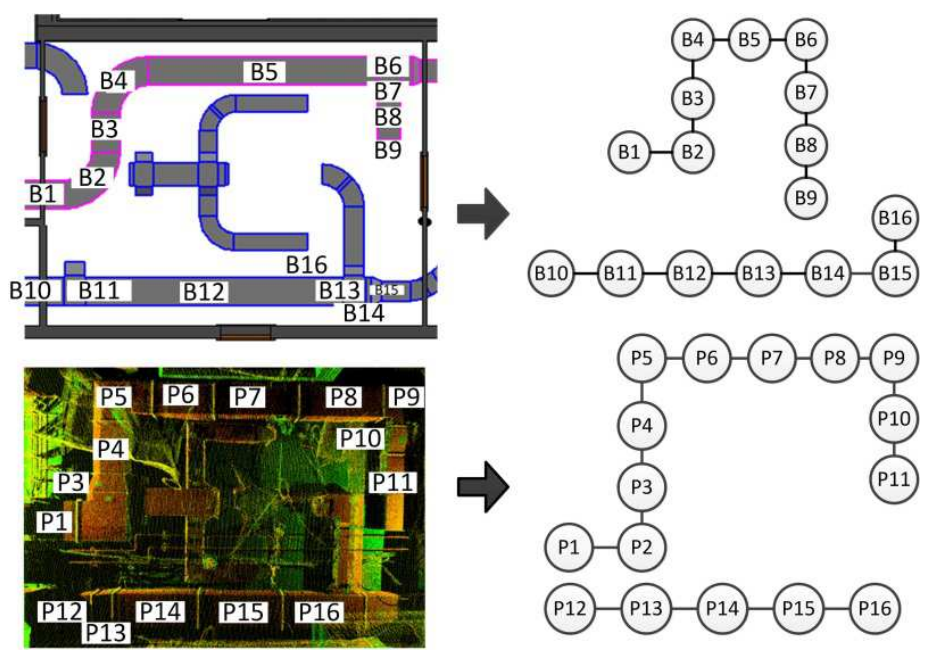

Figure 5 - The topological relationships of components in a point cloud and a BIM 
In a graph structure, a node represents a segment of the point cloud or a component modeled in the BIM. An edge represents the connectivity between the two nodes. The topology-based mapping approach reasons with the graph structures extracted from the point cloud and the BIM, and maps the two nodes together when they have the same topological relationship. For instance, $\mathrm{B}_{2}$ and $\mathrm{P}_{2}$ are the nodes at the corner of the graphs (i.e., the adjacent edges for the node are in two different directions), hence $\mathrm{B}_{2}$ and $\mathrm{P}_{2}$ should be mapped together.

\section{Mapping results analysis}

The mapping results of the three different mapping approaches are evaluated based on the two metrics: precision and recall. The formula to calculate the precision and recall are shown as follows:

$$
\begin{gathered}
\text { Precision= Correct identified mappings } / \text { Total identified mappings } \\
\text { Recall= Correct identified mappings } / \text { Total correct mappings }
\end{gathered}
$$

The correct mappings between segments from a point cloud and components modelled in an asdesigned BIM is identified based on the manual assessment and served as the baseline to evaluate the mapping results gained from the three different mapping approaches. Table 1 shows the mapping results for 16 HVAC ductworks constructed in the case study generated by the three different mapping approaches. As shown in Table 1, different mapping approaches reason with various features (e.g., location, dimension, and topological relationship) of point clouds and BIMs, and generate different mapping results. For the spatial proximity-based mapping approach, one segment in the point cloud might be spatially overlapped with multiple components in the BIM due to discrepancies (e.g., location, shape and dimension discrepancies). For the shape similarity-based mapping approaches, the precision and recall are low due to location and composition discrepancies and the fact that some of the HVAC duct works have the similar shape. Hence, additional features need to be reasoned about in order to eliminate the incorrect mappings. When combining the spatial proximity-based and the shape similarity-based mapping approaches, it is possible to further filter out incorrect mappings solely identified from spatial proximity-based mapping approach. As shown in Table 1, combining the spatial proximity and shape similarity, the precision of the mapping is improved from $75 \%$ to $100 \%$, comparing to the spatial proximity-based mapping approach. However, combing different features together might not always improve the mapping results. In this example, the recall of the mapping is reduced from $80 \%$ to 33\%. The reason for the decrease of the recall is that the combined approach (spatial proximity + shape similarity) is sensitive to the shape discrepancies between the point cloud and the BIM. Hence, this approach intends to remove the mappings when the two mapped components have different shapes even though they are the same component. The topology-based mapping approach is sensitive to the composition and content discrepancies. For instance, when a component $P_{j}$ captured by a laser scan is modeled as a group of components $\left(B_{i 1}, B_{i 2}, B_{i 3}\right)$ in the asdesigned BIM, the topology-based mapping approach is not able to correctly map $\mathrm{P}_{\mathrm{j}}$ to $\mathrm{B}_{\mathrm{i} 1}, \mathrm{~B}_{\mathrm{i} 2}, \mathrm{~B}_{\mathrm{i} 3}$ due to the change of the topology relationship.

Table 1 - The mapping results of the three different mapping approaches

\begin{tabular}{lcc}
\hline Mapping algorithm & Precision & Recall \\
\hline Spatial proximity & $75 \%$ & $80 \%$ \\
Shape similarity & $13 \%$ & $44 \%$ \\
Spatial proximity + shape similarity & $100 \%$ & $33 \%$ \\
Topology & $81 \%$ & $81 \%$ \\
\hline
\end{tabular}

To summarize the findings from the case study, a single mapping approach could not derive all the correct mappings. Instead, when multiple features are combined, it is possible to reduce the impacts of discrepancies on the mapping process and remove incorrect mappings found solely by one single mapping approach or one single feature. 


\section{CONCLUSION AND FUTURE WORK}

BIMs can be used as an information repository to store and deliver as-built information to owners or the facility management teams at the completion of building projects. However, due to discrepancies existing between an as-designed BIM and actual building conditions, the as-designed BIM need to be updated to reflect the as-built conditions. Point cloud data, which captures the actual building conditions, can be mapped to the as-designed BIM in order to facilitate the process of updating the as-designed BIM into the as-built BIM. As the case study shown, different mapping approaches reason with various features of point clouds and BIMs and generate different mapping results. A single mapping approach could not derive all the correct mappings between a point cloud and a BIM. When reasoning with multiple features of point clouds and BIMs, it is possible to improve the mapping result.

Therefore, in order to address the challenge of mapping point clouds to as-designed BIMs, the following research tasks need to be accomplished: (a) identifying different types of features that contribute in recognizing the correspondences between a point cloud and an as-designed BIM; (b) evaluating the mapping results gained by different features; and (c) developing a formalism to combine multiple features together in order to improve the precision and recall for the mapping process. These research tasks are the initial steps towards to a framework that supports the updating of an as-designed BIM frequently throughout a construction or a renovation project by incorporating geometric information captured by progressive laser scans.

\section{REFERENCES}

Dickinson, J., A. Pardasani, S. Ahamed and S. Kruithof (2009). "A survey of automation technology for realising as-built models of services". 1 st International Conference on Improving Construction and Use Through Integrated Design Solutions, CIB IDS365-381.

East, W. and W. Brodt (2007). "BIM for construction handover." Journal of Building Information Modeling: 28-35.

Fallon, K. K. and M. E. Palmer (2007). "General Buildings Information Handover Guide." Principles, Methodology and Case Studies (NISTIR 7417), August.

Gallaher, M. P. and R. E. Chapman (2004). "Cost analysis of inadequate interoperability in the US capital facilities industry", US Department of Commerce, Technology Administration, National Institute of Standards and Technology.

Gao, T., B. Akinci, S. Ergan and J. Garrett (2012). "Constructing of Accurate and Complete As-is BIMs from progressive laser scan data." Gerontechnology 11(2): 75.

O'Brien, J. J. (1998). Construction Change Orders: Impact, Avoidance, and Documentation, McGraw-Hill Professional Publishing.

Pettee, S. R. (2005). "As-builts-Problems \& Proposed Solutions." Construction Management Association of America.

Tang, P., D. Huber, B. Akinci, R. Lipman and A. Lytle (2010). "Automatic reconstruction of as-built building information models from laser-scanned point clouds: A review of related techniques." Automation in construction 19(7): 829-843.

Terwiesch, C. and C. H. Loch (1999). "Managing the process of engineering change orders: the case of the climate control system in automobile development." Journal of Product Innovation Management 16(2):

$160-172$. 